Karstenia 45: 51-61, 2005

\title{
Macrofungi in fire alleys in old quarters of Nurmes, Eastern Finland
}

\author{
KATRI KOKKONEN
}

\begin{abstract}
KOKKONEN, K. 2005: Macrofungi in fire alleys in old quarters of Nurmes, Eastern Finland. - Karstenia 45: 51-61. Helsinki. ISSN 0453-3402.

This paper describes diversity of macrofungi in an old urban environment in Finland. Macrofungi were recorded in fire alleys amidst an old wooden house milieu in the town of Nurmes. The study of one mushroom season revealed a rich mycoflora: 208 species were found, including several rare ones. Location on an esker, along with conservative treatment, likely explains the high species richness.
\end{abstract}

Key words: macromycetes, diversity, fire alleys, urban ecology

Katri Kokkonen, Mahalanniementie 150, FI-75700 Valtimo, Finland

\section{Introduction}

Old wooden house quarters are interesting environments. There are verges, gardens and small parks, which may have been relatively untouched. Botanical studies in such environments in Finland have revealed diverse flora and more uncommon species compared with modern urban environments (e.g. Majuri 1996). Urban macrofungi are more poorly documented than plants. Niemelä and Erkkilä $(1983,1986)$ have studied polypores in the city of Helsinki extensively, and saprophytes on trees have been mapped out in the city centre of Turku (Blomqvist \& Häkkilä 1995). Still, the only reports of urban terrestrial agarics in Finland are from the Botanical Garden of Helsinki (Korhonen 1983), from a nearly virgin wood in the suburban Helsinki (Kotiranta \& Saarenoksa 1987), and from southeastern Finland at the end of the 19th century (Thesleff 1920). In some other European countries, especially in Poland and Holland, urban agarics have been studied better. The recent studies deal with diversities of macrofungi in urban parks, centres of towns, or roadside verges (Stasińska 1994, Keizer \& Arnolds 1995, Łuszczyński 1997, Adema 1999, Savino et al. 1999). Urban mycofloras have turned out to be surprisingly rich.
In this study macrofungi were recorded in the fire alleys within the old wooden house quarters of a small Finnish town during one mushroom season. The back streets of my childhood invited me to explorations. I was delighted. So many various agarics showed up. Possible reasons for the observed high species richness are thoroughly discussed.

\section{History of study area}

The history of the town of Nurmes is short. The small country town was founded around a sound and on an esker between two lakes in 1879. At that time the area was nearly uninhabited having only three estates and a brickyard. Only in 1891 building activities reached the highest parts of the esker and the area of present-day Wooden Nurmes (Puu-Nurmes): the old wooden house quarters of a cultural historical value and my study area. The brickyard, which was situated next to these quarters, was closed in 1892. Most of the lots on the southwest side of the esker were built between 1891 and 1910, whereas on the northeast side between 1900 and 1930. Fire alleys (palosolat) were placed within the quarters for the 
safety from fires. For the same reason, streets were made wide, lots were large and birches were planted along streets and fire alleys. (Saloheimo 1953, Haapio et al. 1976.)

Basically, fire alleys have always been waste land. They have been without systematical care and retained their original character. People have made paths in them, sometimes mowed and fertilized them by releasing garden or other waste products. Sheep and pigs were kept in outbuildings beside fire alleys, and some residents even cultivated potatoes in fire alleys as late as in the 1940s. (Kettunen 1994, R. Kokkonen pers. comm.)

\section{Description of study area}

Nurmes is situated in a transition zone between the southern and the middle boreal vegetation zones in North Karelia, Eastern Finland $\left(63^{\circ} 33^{\prime} \mathrm{N}\right.$, $29^{\circ} 08^{\prime} \mathrm{E}$ ). The annual mean temperature is about $+1.9^{\circ} \mathrm{C}$ and the average precipitation is about 570 $\mathrm{mm}$ per year. The old wooden house quarters belong to the centre of the town. They are situated on an esker and a cape between Lake Pielinen and Lake Nurmesjärvi. The fire alleys run within the quarters, which are in squares.

All the four main fire alleys were studied. Each of them starts from the street on top of the esker and runs 150 metres down the slope to the street below. Two of the fire alleys are situated on the southwest side of the esker, and the other two opposite to them on the northeast side. The fire alleys are 6-9 metres wide. Consequently, the total study area is about 0.5 hectares. The fire alleys are bounded by fences or outbuildings to back yards or exceptionally there is no boundary.

The vegetation varies to some extent among and within the fire alleys. Generally speaking, the vegetation is meadow or grassland with scattered trees. The undergrowth consists of herbs, grasses and mosses. It varies in height a lot from lawns to thickets. The moss layer is usually thin and also spots of bare soil occur. Paths and their surroundings are mowed or trampled occasionally, in some locations more often than in others. Few patches around pines resemble trampled esker forest.

The flora is rather diverse. The dominant vascular plants are Urtica dioica, Anthriscus sylvestris, Glechoma hederacea and Trifolium repens. Ornamental plants are common. Betula is by far the most common tree. Both Betula pendula and Betula pubescens occur. Other tree species in addition to Pinus sylvestris are Salix caprea, Populus tremula, Prunus padus, Acer platanoides, Tilia cordata, Sorbus aucuparia and Picea abies. Almost all Betula and Pinus trees are old, at least 70 years, whereas the others are usually seedlings or young trees. Few old bushes of Sambucus racemosa occur. Further, trees in adjacent back yards, as a Larix sibirica, enrichen the mycoflora. There is hardly any rotten wood on the ground apart from some stumps of birch.

The undergrowth on the southwest side (Fire alleys A and B) is generally richer than on the northeast side. Apparently, this results from a warmer exposition and a longer period of settlement beside Fire alleys A and B. Also, both of these fire alleys have a long row of birches, whereas the others are almost treeless (in november 2003, after my study, nearly all birches were unfortunately felled in Fire alley A). Fire alley A is the most shady one (Fig. 1). It seems to have the most diverse and richest flora. Myosotis sylvatica and Chelidonium majus grow commonly among the previously mentioned dominant plants. In addition to Betula there occur Pinus, Prunus, Acer and Sorbus. No conifers occur in or near Fire alley B. Its tree species are Betula, Salix, Prunus, Acer and Sorbus. There are more mossy lawns than in the other fire alleys. In Fire alley C (Fig. 2), few trees are Pinus, Betula, Salix and Prunus. A common herb is Achillea millefolium. The utmost end of Fire alley $\mathrm{C}$ is the largest patch of pines. In Fire alley $\mathrm{D}$ the vegetation indicates more meagre soil than in the other fire alleys. Rumex acetosella is common. All the possible tree species occur there, but mostly as tiny seedlings.

The bedrock of the study area consists of granite and gneiss (Simonen 1987). The esker is of Quaternary glacifluvial origin and consists of sand and gravel (Kujansuu \& Niemelä 1984). With the naked eye the soil commonly resembles a mixture of sand and mull.

\section{Materials and methods}

Macrofungi were searched for on 20 June, 10 July, and from 9 August to 15 October every or every other week in 2003. Because of the drought at the end of July the searches were continued only in August. Then, the mushroom season was better than usual. I examined paths and other areas of low vegetation and usually avoided thickets. All fruitbodies were considered. Unidentified or otherwise interesting fungi were collected and dried. For the 
identification of species I used microscope, a wide selection of literature available at the Universtity of Turku, and specimens at the Herbaria of the Universities of Turku and Helsinki. Furthermore, other mycologists identified part of my specimens. Arne Aronsen identified some $M y$ cena specimens and Hydropus scabripes, Anton Hausknecht all Conocybe specimens, Ilkka Kytövuori several Cortinarius specimens, Juhani Ruotsalainen some Russula specimens, and Jukka Vauras several Inocybe specimens. Mikael Jeppson confirmed Bovista aestivalis and Lycoperdon lambinonii, and Ilkka Kytövuori Lepiota castanea and $L$. oreadiformis. Collected specimens are deposited at the Herbarium of the University of Turku (TUR). When characteristics of some species are later described, spore size is a range of 10 spores rounded off to $0.5 \mu \mathrm{m}$.

A sample of soil was taken from each fire alley to analyze quantities of nutrients, values of $\mathrm{pH}$ and soil type. The samples were taken to the depth of $10 \mathrm{~cm}$ from typical spots favoured by fungi. The samples were analyzed by Viljavuuspalvelu Ltd. Only one sample per fire alley was analyzed because of high expenses.

The Jaccard similarity coefficients (the number of shared species divided by all species observed at the sites being compared) were calculated to compare similarities of mycofloras between different fire alleys.

\section{Results}

In the fire alleys 208 species of macrofungi were found with certainty (Table 1). Of them, as many as 184 species were agaricales or boletales, 8 gasteromycetes, and only 16 other macrofungi. Many agaricales were difficult to identify. Especially several specimens of Cortinarius were left unidentified.

Table 1. Macrofungi found in the observed fire alleys (A-D). Some taxonomical and ecological notes are given.

\begin{tabular}{llllllllll}
\hline & A & B & C & D & Notes
\end{tabular}

\begin{tabular}{|c|c|c|c|c|c|}
\hline \multicolumn{6}{|l|}{ Agaricales and Boletales } \\
\hline Agaricus silvaticus Schaeff. & & & $\times$ & & \\
\hline Agrocybe molesta (Lasch) Singer & & & $x$ & & \\
\hline Amanita muscaria (L. : Fr.) Hook. & $x$ & $x$ & $x$ & $x$ & \\
\hline Amanita vaginata (Bull. : Fr.) Vittadini & & $x$ & & & \\
\hline Armillaria borealis Marxm. \& Korhonen & $\times$ & $x$ & & & \\
\hline Armillaria cepistipes Velen. & & $x$ & & & \\
\hline Baeospora myosura (Fr. : Fr.) Singer & & & $x$ & & \\
\hline Bolbitius titubans (Bull. : Fr.) Fr. & & & & $x$ & \\
\hline Calocybe carnea (Bull. ex Pers. : Fr.) Donk & & & $x$ & $x$ & \\
\hline Calocybe fallax (Sacc.) Readhead \& Singer & & & $x$ & & \\
\hline Chalciporus piperatus (Bull. : Fr.) Bataille & & $x$ & $x$ & & \\
\hline Clitocybe candicans (Pers. : Fr.) Kumm. & & & & $x$ & \\
\hline Clitocybe clavipes (Pers. : Fr.) Kumm. & & & $x$ & $x$ & \\
\hline Clitocybe fragrans (With. : Fr.) Kumm. & $x$ & & $x$ & $x$ & \\
\hline Clitocybe cf. lohjaënsis Harmaja & & & $x$ & $x$ & 1 \\
\hline Clitocybe menthiodora Harmaja & $x$ & $x$ & & $x$ & 2 \\
\hline Clitocybe metachroa (Fr.) Kumm. & & & $x$ & $x$ & \\
\hline Clitocybe nebularis (Batsch. : Fr.) Kumm. & $x$ & $x$ & $x$ & $x$ & \\
\hline Clitocybe odora (Bull. : Fr.) Kumm. & & $x$ & & & \\
\hline Clitocybe cf. phyllophila (Pers. : Fr.) Kumm. & $x$ & & & & 3 \\
\hline Clitocybe sinopica (Fr. : Fr.) Kumm. coll. & & & $x$ & & \\
\hline Clitocybe squamulosa (Pers. : Fr.) Kumm. & $\times$ & & & & \\
\hline Clitocybe sp. subsect. Epruinatae & & & $x$ & & \\
\hline Collybia butyracea (Bull. : Fr.) Kumm. & $x$ & & $x$ & & \\
\hline Collybia cirrata (Pers.) Kumm. & $x$ & $x$ & $x$ & $x$ & \\
\hline Collybia cookei (Bres.) J.D.Arnold & & $x$ & & & \\
\hline Collybia tuberosa (Bull. : Fr.) Kumm. & & & & $x$ & \\
\hline Collybia sp. & & & $x$ & & \\
\hline
\end{tabular}




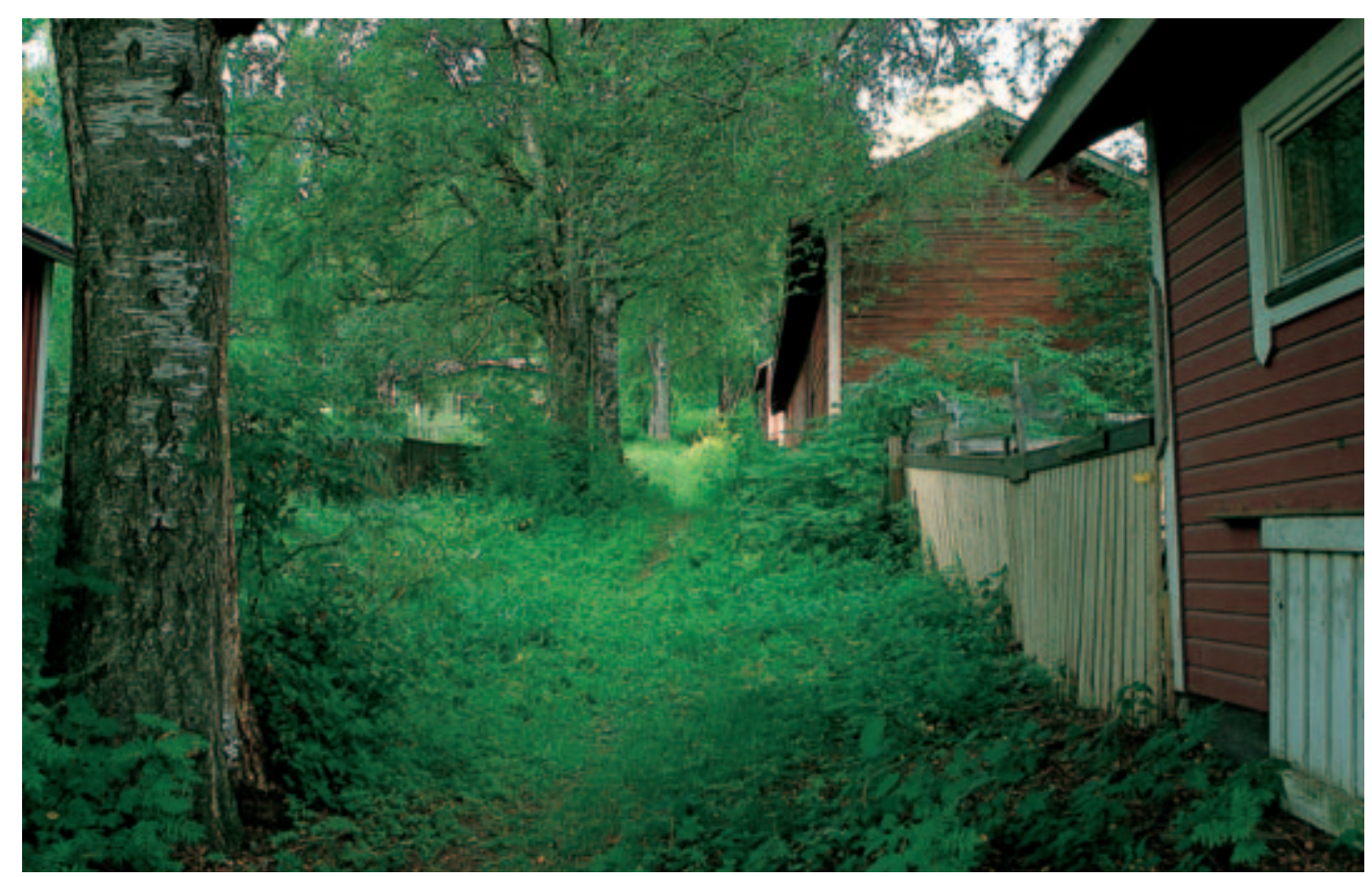

Fig. 1. The fire alley A shaded by birches and having a rich undergrowth on the southwest side of the esker. August 2003, photo: Katri Kokkonen.

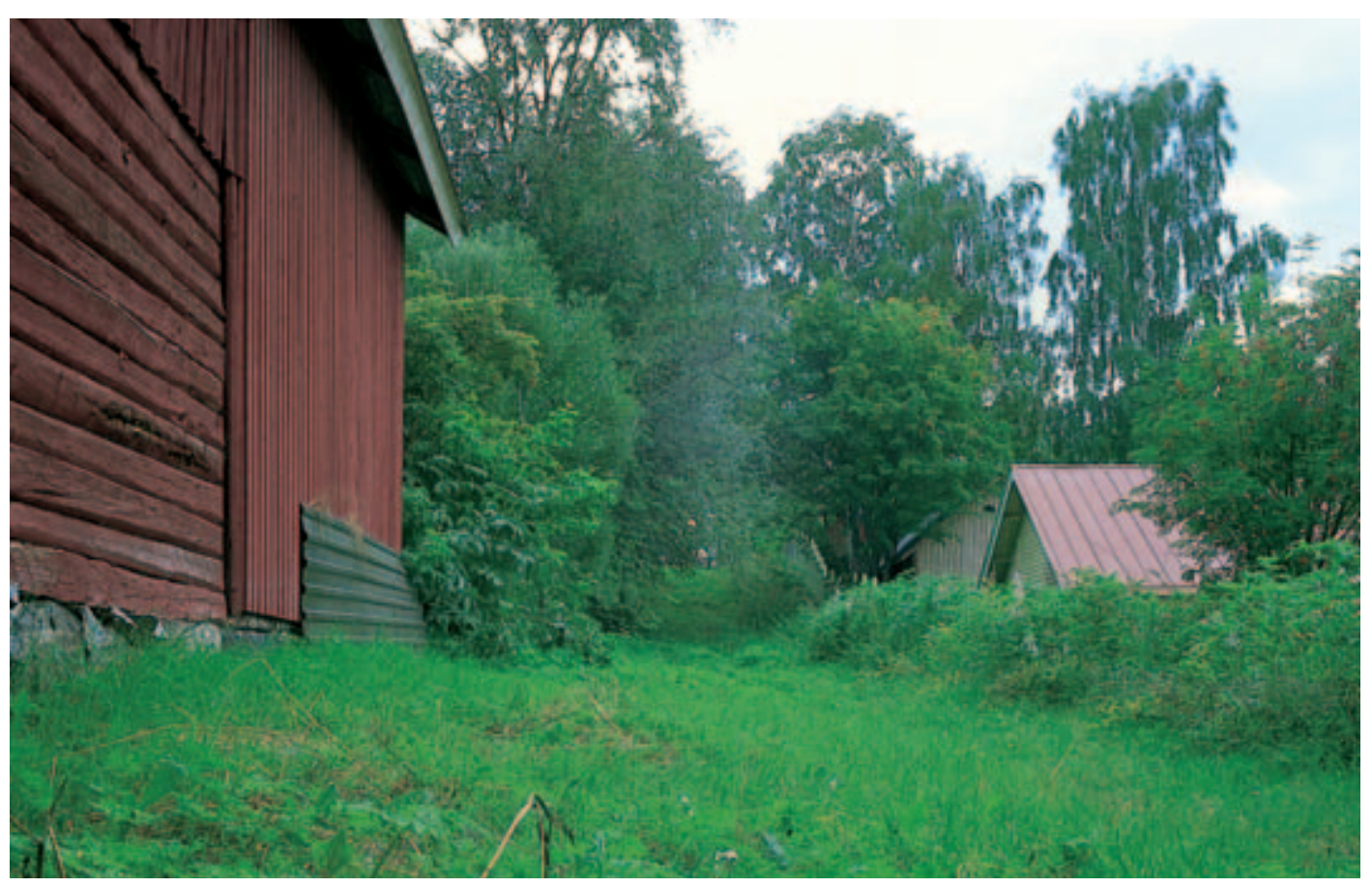

Fig. 2. The mostly open fire alley $\mathrm{C}$ on the northeast side of the esker. August 2003, photo: Katri Kokkonen. 


\begin{tabular}{|c|c|c|c|c|c|}
\hline & A & $\mathrm{B}$ & $\mathrm{C}$ & $\mathrm{D}$ & Notes \\
\hline Conocybe apala (Fr. : Fr.) Arnolds & $x$ & & & & \\
\hline Conocybe echinata (Velen.) Singer & & $x$ & & $x$ & \\
\hline Conocybe mesospora Kühner \& Watling & $x$ & & & & \\
\hline Conocybe rickeniana Orton & & $x$ & & & \\
\hline Conocybe sienophylla (Berk. \& Broome) Singer ss. auct. & $x$ & & & & \\
\hline Conocybe teneroides (Lange) Singer & & & $x$ & & \\
\hline Coprinus comatus (Müll. : Fr.) Pers. & & $x$ & & & \\
\hline Coprinus xanthothrix Romagn. & $x$ & & & & \\
\hline $\begin{array}{l}\text { Cortinarius aureovelatus E. Bendiksen, K. Bendiksen \& } \\
\text { Lindström, ined. }\end{array}$ & & & $x$ & & \\
\hline Cortinarius cf. casimiri (Velen.) Huijsman & & & $x$ & & 4 \\
\hline Cortinarius cinnamomeus (L. : Fr.) Fr. & & & $\times$ & & \\
\hline $\begin{array}{l}\text { Cortinarius cf. diasemospermus Lamoure } \\
\text { var. leptospermus Lindstr. }\end{array}$ & & & & $x$ & \\
\hline Cortinarius hemitrichus (Pers. : Fr.) Fr. & $x$ & $x$ & $x$ & $\hat{x}$ & \\
\hline Cortinarius parvannulatus Kühner coll. & & & $x$ & & \\
\hline Cortinarius cf. parvannulatus Kühner & $x$ & $x$ & & & \\
\hline Cortinarius porphyropus (Alb. \& Schwein.) Fr. & & $x$ & & & \\
\hline Cortinarius saniosus (Fr.) Fr. & & $x$ & $x$ & $x$ & \\
\hline Cortinarius triumphans Fr. & & $x$ & & & \\
\hline Cortinarius umbrinolens Orton & & $x$ & & & \\
\hline Cortinarius vernus Lindstr. \& Melot & & $x$ & & & \\
\hline Cortinarius sp. sect. Vibratilis & & & & $x$ & \\
\hline Cortinarius spp. subgen. Telamonia & $x \times x$ & $x \times x$ & $x \times x$ & $x \times x$ & 5 \\
\hline Cystoderma adnatifolium (Peck) Harmaja & & & $\times$ & & \\
\hline Cystoderma carcharias (Pers.) Konrad \& Maubl. & & & & $x$ & \\
\hline Cystoderma granulosum (Batsch. : Fr.) Kühner & & & & $x$ & \\
\hline Entoloma clypeatum (L. : Fr.) Kumm. & $x$ & & & & \\
\hline Entoloma $\mathrm{cf}$. majaloides Orton & $x$ & & $x$ & & darker umbo \\
\hline Entoloma cf. myrmecophilum (Romagn.) Moser & $x$ & $x$ & & & \\
\hline Entoloma rubrobasis Noordel. & & $x$ & & & 7 \\
\hline Entoloma cf. sericeum (Bull.) Quél. & & & & $x$ & smell absent \\
\hline Entoloma sordidulum (Kühner \& Romagn.) Orton & & & $x$ & $x$ & \\
\hline Entoloma tjallingiorum Noordel. & & & $x$ & & \\
\hline Entoloma undatum (Gill.) Moser & & $x$ & $x$ & & \\
\hline Entoloma sp. 1 & $x$ & & & & \\
\hline Entoloma sp. 2 & $x$ & & $x$ & & \\
\hline Entoloma sp. 3 & & $x$ & & $x$ & \\
\hline Galerina marginata (Batsch.) Kühner & $x$ & & & & \\
\hline Galerina stylifera (Atk.) Smith \& Singer & $x$ & & $x$ & & \\
\hline \multicolumn{6}{|l|}{ Galerina vittiformis (Fr.) Singer var. vittiformis } \\
\hline f. tetraspora Smith \& Singer & & & $x$ & $x$ & \\
\hline Hebeloma helodes Favre & & & & $x$ & \\
\hline Hebeloma cf. helodes Favre & & & $x$ & & \\
\hline Hebeloma leucosarx Orton & & & & $x$ & \\
\hline Hebeloma mesophaeum (Pers.) Quél. & & & $x$ & $x$ & \\
\hline Hebeloma remyi Bruchet ex Quadr & & & $x$ & & \\
\hline Hebeloma sp. sect. Denudata & & & & $x$ & \\
\hline Hemimycena sp. & & & $x$ & $x$ & \\
\hline Hydropus scabripes (Murrill) Singer & & & $x$ & $x$ & \\
\hline Hygrocybe conica (Scop. : Fr.) Kumm. & & & & $\times$ & \\
\hline Hypholoma sp. & & $x$ & & & \\
\hline
\end{tabular}




\begin{tabular}{|c|c|c|c|c|c|}
\hline & A & B & $\mathrm{C}$ & $\mathrm{D}$ & Notes \\
\hline Inocybe calospora Quél. & $x$ & & & & \\
\hline \multicolumn{6}{|l|}{ Inocybe geophylla (Fr. : Fr.) Kumm. } \\
\hline var. geophylla & $x$ & & & & \\
\hline var. lateritia Lange & $x$ & $x$ & & $x$ & \\
\hline var. lilacina (Peck) Gillet & $x$ & & $x$ & & \\
\hline Inocybe grammata Quél. & $x$ & & & & \\
\hline Inocybe lacera (Fr.) Kumm. & & $x$ & $x$ & $x$ & \\
\hline Inocybe leiocephala Stuntz & & & $x$ & & \\
\hline Inocybe mixtilioides Kuyper ad int. & & & & $x$ & \\
\hline Inocybe mixtilis Britzelm. & & & $x$ & $x$ & \\
\hline Inocybe praetervisa Quél. coll. & & $x$ & & $x$ & \\
\hline Inocybe sindonia (Fr.) P.Karst. & & $x$ & & & \\
\hline $\begin{array}{l}\text { Inocybe pseudoasterospora Kühner \& Boursier } \\
\text { var. microsperma Kuyper \& Keizer }\end{array}$ & & & $x$ & & \\
\hline Inocybe sp. sect. Fibrillosae & & & & $x$ & \\
\hline Inocybe sp. 1 sect. Tardae & $x$ & $x$ & & $x$ & \\
\hline Inocybe sp. 2 sect. Tardae & & $x$ & & & \\
\hline Kuehneromyces mutabilis (Schaeff. : Fr.) Singer \& Smith & & $x$ & & & \\
\hline Laccaria laccata (Scop. : Fr.) Berk. \& Broome & $x$ & $x$ & $x$ & $x$ & \\
\hline Laccaria proxima (Boud.) Pat. & & $x$ & & & \\
\hline Laccaria tortilis (Bolton) Cooke & & & $x$ & & \\
\hline Lactarius glyciosmus (Fr. : Fr.) Fr. & & $x$ & $x$ & $x$ & \\
\hline Lactarius rufus (Scop. : Fr.) Fr. & & & $x$ & & \\
\hline Lactarius torminosus (Schaeff. : Fr.) Pers. & & & & $x$ & \\
\hline Lactarius turpis (Weinm.) Fr. & $x$ & $x$ & $x$ & $x$ & \\
\hline Lactarius vietus (Fr.) Fr. & & & $x$ & & \\
\hline Leccinum holopus (Rostk.) Watling & & & & $x$ & \\
\hline Leccinum scabrum (Bull. : Fr.) Gray & & $x$ & $x$ & $x$ & \\
\hline Lepiota castanea Quél. & & $x$ & & & \\
\hline Lepiota cristata (Bolton : Fr.) Kumm. & $x$ & $x$ & & & \\
\hline Lepiota oreadiformis Velen. & $x$ & & & & \\
\hline Lepiota ventriosospora D.A.Reid & & & $x$ & & \\
\hline Lepista irina (Fr.) Bigelow & $x$ & & & & \\
\hline Lepista nuda (Bull. : Fr.) Cooke & $x$ & & $x$ & & \\
\hline Lyophyllum connatum (Schum. : Fr.) & & $x$ & & & \\
\hline Lyophyllum gangraenosum (Fr.) Gulden & & & $x$ & $x$ & \\
\hline Lyophyllum rancidum (Fr.) Singer & & $x$ & $x$ & & \\
\hline Lyophyllum sp. & & $x$ & & & \\
\hline Marasmius epiphyllus (Pers. : Fr.) Fr. & & & & $x$ & \\
\hline Marasmius oreades (Bolt. : Fr.) Fr. & & & $x$ & $x$ & \\
\hline Marasmius rotula (Scop. : Fr.) Fr. & $x$ & $x$ & & & \\
\hline Marasmius scorodonius (Fr. : Fr.) Fr. & $x$ & $x$ & & & \\
\hline Melanoleuca cf. cognata (Fr.) Konr. \& Maubl. & $x$ & & & $x$ & 8 \\
\hline Melanoleuca sp. 1 & $x$ & & & & \\
\hline Melanoleuca sp. 2 & & & & $x$ & \\
\hline Mycena abramsii (Murrill) Murrill & & & $x$ & & \\
\hline Mycena capillaripes Peck & $x$ & & & & \\
\hline Mycena citrinomarginata Gillet & $x$ & $x$ & & & \\
\hline Mycena filopes (Bull. : Fr.) Kumm. & $x$ & $x$ & $x$ & $x$ & \\
\hline Mycena flavoalba (Fr.) Quél. & & & $x$ & $x$ & \\
\hline Mycena galericulata (Scop. : Fr.) Gray & $x$ & & & & \\
\hline Mycena galopus (Pers. : Fr.) Kumm. var. leucogala Cooke & & $x$ & $x$ & & \\
\hline
\end{tabular}




\begin{tabular}{|c|c|c|c|c|c|}
\hline & A & B & $\mathrm{C}$ & $\mathrm{D}$ & Notes \\
\hline Mycena leptocephala (Pers. : Fr.) Gillet & $x$ & $x$ & $x$ & $x$ & \\
\hline Mycena metata (Fr.) Kumm. & $x$ & $x$ & & & \\
\hline Mycena mirata (Peck) Sacc. & $x$ & $x$ & & $x$ & \\
\hline Mycena pura (Pers. : Fr.) Kumm. & $x$ & $x$ & $x$ & $x$ & \\
\hline Mycena rorida (Fr. : Fr.) Quél. & $x$ & $x$ & & & \\
\hline Mycena speirea (Fr. : Fr.) Gillet & $x$ & $x$ & & & one on soil \\
\hline Mycena stylobates (Pers. : Fr.) Kumm. & $x$ & $x$ & & & \\
\hline Mycena tristis Maas. G. & & & $x$ & & 9 \\
\hline Mycena viridimarginata P.Karst. & & $x$ & & & on a rotten plank \\
\hline Mycena sp.1 & & & $x$ & & \\
\hline Mycena sp. 2 & & $x$ & & & \\
\hline Naucoria amarescens Quél. & $x$ & & & & 10 \\
\hline Naucoria bohemica Velen. & $x$ & $x$ & $x$ & $x$ & \\
\hline Panaeolus foenisecii (Pers. : Fr.) Kühner & & $x$ & $x$ & & \\
\hline Panaeolus fimicola (Fr.) Quél. & & & $x$ & & \\
\hline Panellus mitis (Pers. : Fr.) Sing. & $x$ & & & & \\
\hline Panellus serotinus (Schrad. : Fr.) Kühner & & & $x$ & & \\
\hline Paxillus involutus (Batsch. : Fr.) Fr. & $x$ & $x$ & $x$ & $x$ & \\
\hline Pholiota alnicola (Fr. : Fr.) Singer & & $x$ & & & \\
\hline Pholiota elegans Jacobsson & & $x$ & & & \\
\hline Pholiota lenta (Pers. : Fr.) Singer & & $x$ & & & \\
\hline Pholiota limonella (Peck) Sacc. & & $x$ & & & \\
\hline Pholiota spumosa (Fr.) Singer & & & & $x$ & on board \\
\hline Pluteus atricapillus (Batsch.) Fayod & $x$ & & & & \\
\hline Psathyrella candolleana (Fr. : Fr.) Maire & & & $x$ & & \\
\hline Psathyrella corrugis (Pers. : Fr.) Konrad \& Maubl. & $x$ & & & & \\
\hline Psathyrella $\mathrm{cf}$. spadicea (Schaeff.) Singer & $x$ & & & & 11 \\
\hline Psathyrella sp. & $x$ & & & & \\
\hline Psilocybe inquilina (Fr. : Fr.) Bres. & & & $x$ & $x$ & \\
\hline Psilocybe sp. & & & $x$ & & \\
\hline Rickenella fibula (Bull. : Fr.) Quél. & & & & $x$ & \\
\hline Rickenella setipes (Fr. : Fr.) Quél. & & & & $x$ & \\
\hline Rhodocybe caelata (Fr.) Maire & & $x$ & & & \\
\hline Rhodocybe hirneola (Fr. : Fr.) Orton & & & $x$ & & \\
\hline Russula aeruginea Lindblad & $x$ & $x$ & $x$ & $x$ & \\
\hline Russula cessans Pearson & $x$ & & $x$ & & \\
\hline Russula claroflava Grove & $x$ & $x$ & & & \\
\hline Russula delica Fr. coll. & & $x$ & & & \\
\hline Russula gracillima Jul.Schäff. & & $x$ & & $x$ & \\
\hline Russula nitida (Pers. : Fr.) Fr. & $x$ & $x$ & $x$ & $x$ & \\
\hline Russula versicolor Jul.Schäff. coll. & & $x$ & & $x$ & \\
\hline Russula xerampelina (Schaeff.) Fr. coll. & $x$ & & $x$ & $x$ & \\
\hline Russula sp. cf. Rhodellinae & $x$ & & & & \\
\hline Simocybe rubi (Berk.) Watling & $x$ & & & & 12 \\
\hline Strobilurus tenacellus (Pers. : Fr.) Singer & $x$ & & & & in August \\
\hline Stropharia cyanea (Bull.) Tuom. & & $x$ & $x$ & & \\
\hline Stropharia percevalii (Berk. \& Broome) Sacc. & & $x$ & $x$ & & \\
\hline Suillus grevillei (Klotzsch : Fr.) Singer & $x$ & & & & \\
\hline Suillus luteus (L. : Fr.) Roussel & & & & $x$ & \\
\hline Tricholoma fulvum (Bull. : Fr.) Bigeard \& Guill. & & $x$ & & $x$ & \\
\hline Tricholoma stiparophyllum (Lund) P.Karst. & $x$ & & & $x$ & \\
\hline Tricholoma imbricatum (Fr. : Fr.) Kumm. & & & $x$ & & \\
\hline
\end{tabular}




\begin{tabular}{|c|c|c|c|c|c|}
\hline & A & $\mathrm{B}$ & $\mathrm{C}$ & $\mathrm{D}$ & Notes \\
\hline Tubaria furfuracea (Pers. : Fr.) Gillet & $x$ & & $x$ & $x$ & \\
\hline Xerocomus fennicus (Harmaja) H.Ladurner \& Simonini & $x$ & $x$ & & & \\
\hline Xerocomus subtomentosus (L. : Fr.) Quél. & & $x$ & $x$ & $x$ & \\
\hline \multicolumn{6}{|l|}{ Gasteromycetes } \\
\hline Bovista aestivalis (Bonord.) Demoulin & $x$ & $x$ & $x$ & & \\
\hline Bovista nigrescens Pers. : Pers. & $x$ & & $x$ & & \\
\hline Crucibulum laeve (Huds.) Kambly & $x$ & & & $x$ & \\
\hline Lycoperdon lambinonii Demoulin & $x$ & & & & \\
\hline Lycoperdon cf. nigrescens (Pers. : Pers.) Pers. & & $x$ & & & \\
\hline Lycoperdon molle Pers. : Pers. & & & $x$ & & \\
\hline Lycoperdon perlatum Pers. : Pers. & $x$ & & $x$ & $x$ & \\
\hline Lycoperdon pyriforme Schaeff. : Pers. & $x$ & & & & \\
\hline \multicolumn{6}{|l|}{ Aphyllophorales } \\
\hline Antrodia serialis (Fr.) Donk & $x$ & & & & on board \\
\hline Auriscalpium vulgare Gray & $x$ & & $x$ & $x$ & \\
\hline Bjerkandera adusta (Willd. : Fr.) P.Karst. & & $x$ & & & \\
\hline Clavulina cristata (Holmsk. : Fr.) J.Schröt. & & $x$ & & & \\
\hline Clavulinopsis luteoalba (Rea) Corner & & & & $x$ & \\
\hline Ganoderma lipsiense (Batsch.) G.F. Atk. & & $x$ & & & \\
\hline Inonotus obliquus (Pers. : Fr.) Pilát & $x$ & & & & \\
\hline Macrotyphula fistulosa (Holmsk. : Fr.) R.H.Petersen & $x$ & $x$ & $x$ & $x$ & \\
\hline Macrotyphula juncea (Alb. \& Schwein. : Fr.) Berthier & & & & $x$ & \\
\hline Phellinus cinereus (Niemelä) M.Fisch. & $x$ & $x$ & & & \\
\hline Polyporus brumalis (Pers. : Fr.) Fr. & $x$ & $x$ & & & \\
\hline \multicolumn{6}{|l|}{ Ascomycetes } \\
\hline Helvella chinensis (Velen.) Nannf. \& L.Holm & $x$ & $x$ & & $x$ & \\
\hline Hymenoscyphus virgultorum (Weber) W.Phillips & & & & $x$ & \\
\hline Nectria cinnabarina (Tode : Fr.) Fr. & $x$ & $x$ & & $x$ & \\
\hline Otidea onotica (Pers. : Fr.) Fuckel & $x$ & $x$ & & & \\
\hline Tarzetta catinus (Holmsk. : Fr.) Korf \& J.K.Rogers & & $x$ & & & \\
\hline
\end{tabular}

1 smell absent at least in old fruitbodies; spores ellipsoid - subgloboid, 4-6 × 3-3.5 $\mu \mathrm{m}$ or in another sample 4-5 $\times 3-4 \mu \mathrm{m}$

2 in Melzer's solution small red brown crystals and droplets on, and possibly within, basidioles and hyphae of cap cuticle

3 cap margin distinctly pruinose, disc not or slightly pruinose; smell absent or slightly radish; spores 4-5 × 2.5$3 \mu \mathrm{m}$

4 spores ellipsoid, rather weakly verrucose, $10-13.5 \times 6-7.5 \mu \mathrm{m}$; near Pinus sylvestris

5 at least 8 species on the basis of spore morphology

6 not pallescent on drying; spores $7-9 \times 6-7.5 \mu \mathrm{m}$

7 stem pale grey, several without red tomentum at the base; pileipellis with scarce or absent brown intracellular pigment and fine encrustations; compared with the type and other specimens at TUR

8 spores fragile breaking easily

9 on needle litter under Pinus sylvestris, nearby Betula

10 on a mossy heap of litter

11 cap 2.7-3 cm; spores 5.5-7.5 × 3.5-4.5 $\mu \mathrm{m}$; at the base of Betula

12 on branches of old Sambucus racemosa 
The most common species were Amanita muscaria, Clitocybe nebularis, Collybia cirrata, Cortinarius hemitrichus, Laccaria laccata, Lactarius turpis, Macrotyphula fistulosa, Mycena filopes, M. leptocephala, M. pura, Naucoria bohemica, Paxillus involutus, Russula aeruginea and Russula nitida. These species occured in every fire alley (Table 1). Other common taxa, as recorded in three fire alleys, were Auriscalpium vulgare, Bovista aestivalis, Clitocybe fragrans, Cortinarius saniosus, Helvella chinensis, Inocybe geophylla var. lateritia, I. lacera, Inocybe sp. 1 sect. Tardae, Lactarius glyciosmus, Leccinum scabrum, Lycoperdon perlatum, Mycena mirata, Nectria cinnabarina, Russula xerampelina coll., Tubaria furfuracea and Xerocomus subtomentosus.

Most species were found rarely, which is a normal result in community studies (Gaston 1994). About half of all species were found only in one fire alley (Table 1). According to the present knowledge, several observed species are regarded as rare in North Karelia: Agrocybe molesta, Calocybe carnea, C. fallax, Clitocybe menthiodora, Conocybe mesospora, C. teneroides, Entoloma clypeatum, E. rubrobasis, E. tjallingiorum, E. undatum, Hydropus scabripes, Inocybe calospora, I. pseudoasterospora var. microsperma, Lepiota castanea, L. oreadiformis, Lepista irina, Lyophyllum gangraenosum, Marasmius rotula, Mycena abramsii, $M$. tristis, Naucoria amarescens, Simocybe rubi, Stropharia percevalii, Xerocomus fennicus, Bovista aestivalis, Clavulinopsis luteoalba, Helvella chinensis and Otidea onotica. Many of them are known to be more common in southern Finland. Entoloma rubrobasis is critically endangered and Bovista aestivalis nearly threatened species in Finland (Rassi et al. 2001). Further, Inocybe calospora and Lepiota castanea are regionally threatened species in the eastern middle boreal zone (www.environment.fi).

Most species, 66\%, were saprophytes. They were predominantly decomposers of litter and soil. Predictably, the vast majority of mycorrhizal species were symbionts of Betula.

The numbers of species did not differ noticeably among the fire alleys (Table 2). However, every fire alley had an unique mycoflora: $31-37 \%$ of the species occurred nowhere else and the Jaccard similarity coefficients were rather low. Mycofloras in Fire alleys C and D (0.31) as well as A and B (0.28) resembled each others most, but still not considerably. Most of the regionally rare species occured in Fire alleys A, B and C (Table 1).

Table 2. Number of species of macrofungi in each fire alley $\left(\mathrm{N}_{\mathrm{t}}\right)$, number of species of macrofungi found only in the fire alley in question $\left(\mathrm{N}_{\mathrm{r}}\right)$, and Jaccard similarity coefficients $(\mathrm{J})$ between mycofloras of different fire alleys A-D.

\begin{tabular}{rrrrrr}
\hline & & A & B & C & D \\
\hline $\mathrm{N}_{\mathrm{t}}$ & & 86 & 89 & 89 & 81 \\
$\mathrm{~N}_{\mathrm{r}}$ & & 32 & 33 & 31 & 25 \\
$\mathrm{~J}$ & $\mathrm{~A}$ & 1.00 & & & \\
& $\mathrm{~B}$ & 0.28 & 1.00 & & \\
& $\mathrm{C}$ & 0.19 & 0.20 & 1.00 & \\
& $\mathrm{D}$ & 0.20 & 0.22 & 0.31 & 1.00 \\
\hline
\end{tabular}

Table 3. Values of $\mathrm{pH}$ and quantities of nutrients in the soil samples from the observed fire alleys (A-D).

\begin{tabular}{lrrrrr}
\hline Fire alley & A & B & C & D \\
\hline $\mathrm{pH}$ & & 6.5 & 5.6 & 5.8 & 5.3 \\
$\mathrm{~K}$ & $\mathrm{mg} / \mathrm{l}$ & 322 & 151 & 92 & 68 \\
$\mathrm{Ca}$ & $\mathrm{mg} / \mathrm{l}$ & 1830 & 872 & 1020 & 837 \\
$\mathrm{Mg}$ & $\mathrm{mg} / \mathrm{l}$ & 196 & 107 & 113 & 61 \\
$\mathrm{P}$ & $\mathrm{mg} / \mathrm{l}$ & 29 & 17 & 23 & 17 \\
\hline
\end{tabular}

The quantities of nutrients and the values of $\mathrm{pH}$ are presented in Table 3. Potassium, phosphorus and $\mathrm{pH}$ varied from sufficient to good in terms of fruitfulness for cultivation. The highest scores of $\mathrm{pH}$ and all nutrients were in the sample from Fire alley A and the lowest in the sample from Fire alley D. Soil type was fine-grained sand rich in mull. In the sample from Fire alley A the sand component was slightly more fine-grained than in other samples.

\section{Discussion}

The fire alleys in the old wooden house quarters of Nurmes have an astonishingly rich mycoflora. In an area of 0.5 hectares 208 species of macrofungi were found in one year. Of them, 192 species were agaricales, boletales or gasteromycetes. However, the comparison is problematic. Studies of urban mycoflora are few and their methods use 
to vary. In a suburb of Helsinki, Kotiranta and Saarenoksa (1987) recorded nearly 360 species of basidiomycetes in an area of 5.7 ha of a rich, nearly virgin wood within several years. Of those species, 260 were agaricales, boletales or gasteromycetes. A more similar habitat with the fire alleys is a 0.8 ha yard and garden of an old farm house in southern Finland, where Heinonen (1997) recorded 187 species of macrofungi in the course of many years. In Holland, Adema (1999) reported as many as 193 species of macrofungi in the old centre of Leiden comprising the botanical garden during several years. He excluded many unfamiliar species to himself. In a Polish recreational park of 23.5 ha Stasińska (1994) recorded 144 species of macrofungi in two years. Rather similarly, Savino et al. (1999) registered 94 and 116 species of macrofungi in two Italian urban parks of 19 and 21 ha during one whole year. No doubt, the species richness in the observed fire alleys is greater than generally in urban parks.

The fire alleys are a rather heterogeneous environment, which increases the number of microhabitats for fungi. The openness and the composition of vegetation, as well as the quantities of nutrients vary to some extent. Elements of various environments also tend to mix with each other in the fire alleys. For above reasons there occur macrofungi typical for meadows, gardens, parks, roadsides, pastures, herb-rich forests and heath forests. Also rare, less known or critical species occurred. Mycofloras of built, enriched or sunbathed slopes of eskers have not been investigated before in Finland.

Some of the found species grow usually on sand or acid soil, while others are indifferent or prefer richer soil. Requirements of several species are poorly known. Possibly a mixture of sand and mull, the common composition of soil in the fire alleys, is suitable or ideal for many species of macrofungi, especially saprophytes. Saprophytes dominated for species number, as is usually the case for enriched and ruderal sites. According to Lange (1993), saprophytes on litter and soil are rather indifferent to soil type, unlike mycorrhizals.

After the initial manipulation of the esker vegetation cautious treatment of the fire alleys has preserved heterogeneity and created stability, which especially rare species often require.

In addition, microclimate may be beneficial between the two lakes and amongst warming set- tlement. That would explain the occurrence of several southern species. Alternatively, manmade habitats may be more poorly examined at the level of North Karelia. Favourable weather conditions in autumn 2003 certainly contributed to the study. In summer, however, few fungi were detected partly because of dryness.

Many of the common species were mycorrhizal symbionts of Betula and occur customarily almost everywere or in parks. Inocybe and Russula species are known to be better adapted to enriched man-made habitats than other mycorrhizals, which is supported also by this study. They were the most diverse genera of mycorrhizals in addition to Cortinarius. Even some rare species of Inocybe occurred. The diversity of Cortinarius, especially of the subgenus Telamonia, was surprisingly high.

Some interesting shared species catch the eye, when different urban or 'semiurban' studies are compared with each others: Marasmius rotula is mentioned in seven out of eight studies including this study, Lepista irina is mentioned in all four, and Entoloma undatum in three Finnish studies, whereas Calocybe carnea is invariably mentioned in foreign urban studies (Korhonen 1983, Kotiranta \& Saarenoksa 1987, Stasińska 1994, Keizer \& Arnolds 1995, Heinonen 1997, Adema 1999, Savino et al. 1999). A rare species occurring usually in pastures was Lepiota oreadiformis in one fire alley. Adema (1999) mentioned Lepiota oreadiformis from the embankment of the foss in Leiden, Holland. Thus, some species of unfertilized grasslands or dry meadows may thrive also in urban areas, as emphasized by Keizer and Arnols (1995) for roadside verges.

However, species of nutrient-poor grasslands were exceptions in the fire alleys. For example rare species of Hygrocybe were absent, as commonly in urban studies. Many of the recorded species prefer rich soil. All the three fire alleys with the richest soil samples had a diverse mycoflora and harboured most of the rare species. On the other hand, the variation in quantities of nutrients and vegetation was probably largest in these fire alleys. Mycofloras might also be undergoing a change into some direction since the clearing. As a result, virtually nothing can be said about the effect of enrichment on the species richness.

The rarely seen Simocybe rubi was found on Sambucus racemosa, while Kotiranta and Saarenoksa (1987) mentioned it on Salix caprea 
in Helsinki. On branches of old Sambucus racemosa grew also interestingly Mycena mirata. Sambucus racemosa has been probably ignored by mycologists. Mycena tristis has been found twice before in Finland from the province of South Häme under Betula, and Hydropus scabripes only once from a protected, calcareous herb-rich forest (Kosonen 1994). The recently described Pholiota elegans has been also formerly recorded mostly from virgin forests (Holec 2001) and Entoloma rubrobasis only from moist sites in forests (J. Vauras pers. comm.). Both occurred in the same fire alley among short grasses and herbs shaded by birches. Also once before I have found Entoloma rubrobasis from a grassland near birches in the neighbouring commune of Valtimo.

The species were evenly distributed to all fire alleys. In other words, the fire alleys complement each others well. Their mycofloras differed rather much from each others even on the same side of the esker, in spite of similar history and exposition. However, the brief duration of the study makes conclusions about distributions questionable.

To sum up, this study illuminates mycoflora in one, mycologically perhaps special urban environment. More information about species, their distributions and ecology is needed.

Acknowledgements: I thank Jukka Vauras for helpful comments, showing literature, and identifying some species. Thanks are extended to Arne Aronsen, Anton Hausknecht, Mikael Jeppson, Ilkka Kytövuori, and Juhani Ruotsalainen for identifications of fungi, Mika Toivonen for notices about Cortinarius, and Seppo Huhtinen for commenting the manuscript and letting me use materials of the Herbarium of the University of Turku. Pertti Salo kindly let me study at the Herbarium of the University of Helsinki. I am very grateful to my family for support.

\section{References}

Adema, J.P.H.M. 1999: Paddestoelen in Leiden. - Coolia 42 (1): 21-31.

Blomqvist, I. \& Häkkilä, S. 1995: Turun ydinkeskustan lahottajasienet. - Unpublished paper for the city of Turku and the Botanical Museum of the University of Turku. 13 pp.

Gaston, K. J. 1994: Rarity. - London. 205 pp.

Haapio, H., Järvisalo, J., Lepäntalo, O., Linnainmaa, A., Lintunen, L., Pailinna, P., Puumalainen, M., Salonen, K., Keiski-Sankari, S., Sederholm, M., Storgårds, H., Suhonen, P., Sund, A., Tukkila, L., Wainikainen, P., Varjoranta, K., Viljakainen, L. \& Virtanen, K. 1976: Puu-Nurmes. Kulttuurihistoriallisesti arvokkaan poh- joiskarjalaisen asuinalueen kehittämissuunnitelma. Publications of the University of Art and Design. Helsinki. 99 pp.

Heinonen, M.-L. 1997: Ympyrä 50 m mökin rappukiveltä. - Sienilehti 3: 69-74.

Holec, J. 2001: The genus Pholiota in central and western Europe. - Libri Botanici 20: 1-220.

Keizer, P.J. \& Arnolds, E. 1995: The Macromycete flora in roadside verges planted with trees in comparison with related forest types. - Mycol. Helv. 7: 31-69.

Kettunen, T. 1994: Puu-Nurmes 1876-1992. Tutkimus kodeista ja arkipäivän elämästä. - Master's thesis. Department of European Ethnology, University of Turku. 114 pp.

Korhonen, M. 1983: Kasvitieteellisen puutarhan suursienistä (Macrofungi in the Botanical Garden of the University of Helsinki). - In: Jalas, J. (ed.), Kasvitieteellisen puutarhan eliömaailmaa 1: 75-82. Helsinki.

Kosonen, L. 1994: Sienilöytöjä Pirkanmaalta: pikarihaarakas, veritatti, hiipponukkalakki ja suruhiippo. Talvikki 1: 39-42.

Kotiranta, H. \& Saarenoksa, R. 1987: Helsingin Kumpulan laakson sienilajistosta 2. Heltta- ja kupusienet. Sienilehti 39: 19-26.

Kujansuu, R. \& Niemelä, J. 1984 (ed.): Maaperä (kartta). - In: Suomen kartasto (Atlas of Finland): 123 126, Geologia. Geologian tutkimuslaitos.

Lange, M. 1993: Macromycetes under twelve tree species in ten plantations on various soil types in Denmark. - Opera Bot. 120: 1-53.

Łuszczyński, J. 1997: Interesting macromycetes found in the Kielce town (Central Poland). - Acta Mycol. 32: 207-228

Majuri, P. 1996: Naantalin vanhankaupungin kasvillisuus. - Unpublished paper for the town of Naantali. 19 pp.

Niemelä, T. \& Erkkilä, R. 1983: Käävät Helsingin yliopiston kasvitieteellisessä puutarhassa (Polypores in the Botanical Garden of the University of Helsinki). In: Jalas, J. (ed.), Kasvitieteellisen puutarhan eliömaailmaa 1: 83-93. Helsinki.

Niemelä, T. \& Erkkilä, R. 1986: Polypores in the parks and forests of the City of Helsinki. - Karstenia 26: 140.

Rassi, P., Alanen, A., Kanerva, T. \& Mannerkoski, I. (toim.) 2001: Suomen lajien uhanalaisuus 2000. Ympäristöministeriö \& Suomen ympäristökeskus, Helsinki.

Saloheimo, V. 1953: Nurmeksen historia. - Kuopio. 867 pp.

Savino, E., Del Frate, G., Boccardi, A. \& Bonazzi, M. 1999: Nota preliminare sui macromiceti dei parchi comunali di Rozzano (MI). - Mic. Ital. 3: 10-17.

Simonen, A. 1987: Kallioperä (kartta). - In: Suomen kartasto (Atlas of Finland): 123-126, Geologia. Geologian tutkimuslaitos.

Stasińska, M. 1994: Macromycetes in a recreational park in Łódź. - Acta Mycol. 29: 229-236.

Thesleff, A. 1920: Studier öfver basidsvampfloran i sydöstra Finland med hänsyn till dess sammansättning, fysiognomi, fenologi och ekologi. - Bidr. Känned. Finlands Nat. Folk 79 (1): 1-140. 